\title{
La obra de Lavoisier como un modelo para la historia de las ciencias
}

\author{
Julio Alejandro Castro Moreno*
}

Artículo recibido: 14-8-2006 y aprobado: 30-4-2008

Lavoisier's work like a model to science history

Resumen: Este artículo muestra algunas características de la historia de las ciencias que consideramos de suma importancia. Dentro de ellas destacamos tres: la discontinuidad que se presenta en la construcción del saber científico, el proceso de diferenciación que tuvo la ciencia entre los siglos XVII y XVIII, y las interrelaciones que se presentan entre sociedad y ciencia. Para poder llevar a cabo nuestro propósito, hemos hecho un análisis de la vida y obra de Antoine Laurent de Lavoisier. Las características mencionadas han sido detalladas en cada uno de los apartes en que se ha subdividido el escrito, pero, como se verá, hay un diálogo permanente entre cada una de ellas.

Palabras clave: Discontinuidad, diferenciación, contexto sociocultural, actividad científica, historia de las ciencias.
Abstract: This article shows three characteristics of science history which are considered as the main ones by the authors of this paper. First, the discontinuity that is possible to find in the processes of knowledge building; second, the process of disciplines differentiation that science suffered along the $17^{\text {th }}$ and $18^{\text {th }}$ centuries; finally, the relationships between science and society. The analysis of Lavoisier's life gives us an important example to study those characteristics, not only in their specific meanings but also considering their mutual relations.

Key words: Discontinuity, differentiation, social and cultural context, scientific activity, science history.

Profesor de la Universidad Pedagógica Nacional, Bogotá, Colombia. jcastro@pedagogica.edu.co. 


\begin{abstract}
Convencido de estas verdades, me he impuesto la ley de no pasar nunca más que de lo conocido a lo desconocido, de no deducir ninguna consecuencia que no se derive inmediatamente de las experiencias y observaciones, y de encadenar los hechos y verdades químicas en el orden más apropiado que facilita la comprensión a los principiantes.
\end{abstract}

Antoine Laurent de Lavoisier, Tratado elemental de química.

\section{Introducción}

En primer lugar, debemos reconocer que el tema que aquí abordamos no es novedoso, ya que se han escrito innumerables trabajos acerca de "la vida y obra de Lavoisier". Sin embargo, también consideramos que este artículo puede ser una innovación en cuanto al tratamiento que le damos al tema, en la medida en que proponemos un modelo para la historia de las ciencias. En ese sentido, también es posible que dicho modelo dé luces acerca de importantes implicaciones didácticas, tanto en el terreno de la enseñanza de las ciencias, como en el de la formación de maestros. Tales implicaciones están íntimamente relacionadas con la elaboración y socialización de estudios histórico-críticos, como el que ahora presentamos.

Los estudios histórico-críticos de las ciencias naturales cada vez están adquiriendo mayor importancia en diferentes ámbitos (el educativo, por ejemplo), sobre todo porque dan cuenta del porqué y el cómo se han establecido las teorías que consideramos válidas y la manera en que dejaron de serlo aquellas que han pasado a la historia. Con este pasar a la historia queremos denotar un hecho que aún sigue presente en algunos discursos históricos: se considera historia a todo aquello que fue y dejó de ser, aquello que en algún momento fue válido para una comunidad y que ahora se considera como disparates o errores del pasado. Desde nuestro punto de vista, la historia es una reconstrucción constante, es formular problemas y proponer soluciones y, por qué no decirlo, es aventurarse a meterse en los zapatos de aquellos que han dedicado su tiempo para que dejemos de ser principiantes y, poco a poco, nos volvamos sabios.

Según las historias tradicionales, en el momento en que algunos colegas de Lavoisier intercedieron por él para evitar que su cabeza rodara, alguien les contestó que la República no necesitaba sabios. Pero, al parecer, esta frase es apócrifa. En todo caso, independientemente de la pronunciación o no de esta frase, queremos mostrar en este escrito que realmente las sociedades sí necesitan a los sabios, y mucho más, que los sabios precisan muchísimo de las sociedades en las cuales viven.

Nuestro proyecto es mostrar algunas características esenciales de la historia de las ciencias, usando para ello la vida y obra de Lavoisier. Estas propiedades que asumimos como fundamentales son las siguientes: la no-diferenciación en los primordios de la ciencia, es decir, la ciencia que se profesaba alrededor de los siglos XVII y XVIII; la discontinuidad en la historia de las ciencias, o sea, que la forma en que se da paso de una teoría a otra ocurre por rupturas o revoluciones y no por aleación o ensamblaje; y, por último, la estrecha relación que se da entre ciencia y sociedad.

Hemos intentado separar estos tres aspectos de la dimensión histórico-crítica de las ciencias, pero, como veremos, no es factible hacer tal separación de un 
modo total. Sin embargo, para efectos de nuestra presentación, asumimos que es posible hacerlo.

\section{Lavoisier como geólogo, físico, químico y biólogo. 0 la no-diferenciación en los primordios de la ciencia}

Es muy interesante notar que antes de que existieran las disciplinas totalmente diferenciadas, como ocurrió a lo largo del siglo XIX, aquellos que emprendían la labor científica lo hacían desde diferentes ramas del saber. Por ejemplo, Stahl fue médico y, sin embargo, se le conoce como químico. Cuando Darwin emprendió su viaje en el Beagle fue contratado como geólogo más que como naturalista', es decir, como biólogo². Aún más, Darwin antes de embarcarse en el mundo de las ciencias naturales había estudiado para médico y para clérigo, aunque no culminó ninguna de estas carreras.

También es interesante ver cómo a partir del siglo XX las ciencias se han superespecializado, volviéndose cada vez más específicas, y han aparecido amalgamas de disciplinas como la biología molecular. En este último ejemplo, podemos notar que vuelve a haber otro tipo de no-diferenciación, en el sentido que en la biología molecular han confluido biólogos, químicos, físicos, médicos e ingenieros, pero lo han hecho desde

1 Puede decirse que algunas rupturas con la historia natural contribuyeron al establecimiento de la biología como ciencia independiente.

2 Aunque en su época y su país no existía una profesión que pudiera denominarse como tal, ya que había varias ramas del saber que estudiaban lo vivo, pero aún no se había consolidado la biología como una ciencia independiente y autónoma. una formación científica claramente especializada, mientras que los científicos de los siglos anteriores al XIX emprendían sus labores investigativas desde diferentes puntos de vista, o sea, desde diversas disciplinas que aún no estaban totalmente cimentadas como las conocemos ahora.

Veamos cómo esto puede ser ejemplificado con Lavoisier: en primer lugar, es necesario resaltar que se graduó como abogado en 1764, siguiendo una tradición familiar, y dos años más tarde emprendió trabajos en geología, bajo la dirección de Guettard. En 1768 entró a ocupar un cargo en la Academia Real de las Ciencias de París, debido a que uno de sus integrantes, el químico Baron, había muerto. Aunque el Rey le dio la vacante al ingeniero Jors, Lavoisier logró hacer parte de esta institución como miembro supernumerario (BensaudeVincent, 1998).

Sin embargo, antes de entrar a hacer parte de la Academia, Lavoisier ingresó a la Ferme Génerale, una institución que recaudaba impuestos para el Estado y ello fue posible porque había recibido una herencia por parte de su abuela. Este tipo de actividad fue muy importante en la vida de Lavoisier y, como veremos en la tercera parte de este escrito, de algún modo, el ser recaudador de impuestos le costó la propia vida.

Retomando la actividad científica de Lavoisier, podemos observar que se hizo químico más por casualidad que por sus propios intereses. Sus primeros trabajos fueron sobre geología y se acercó a la química como una ciencia auxiliar de ésta, y cuando hubo la vacante en la Academia, él pidió que se creara una para física experimental, ya que se describía a 
sí mismo como físico, debido a su acercamiento a las ciencias experimentales y su distanciamiento de las que privilegiaban la observación, como la geología (Bensaude-Vincent, 1998).

Finalmente, podemos ver a Lavoisier como uno de los personajes más importantes en el desarrollo de la química, de ahí que relacionemos su nombre al de esta ciencia. Sus trabajos en dicha disciplina serán detallados a lo largo de este escrito, pero nos falta mencionar una faceta científica de Lavoisier: la de biólogo. Obviamente, cuando hablamos de Lavoisier como biólogo o químico no estamos aludiendo al significado actual de estas profesiones. Lavoisier era un científico, pero la ciencia de su época pasaba por un proceso de diferenciación que en muchos casos apenas estaba iniciando, por ejemplo, la biología, mientras que la física estaba más cimentada como una ciencia con un objeto de estudio claramente especificado.

Si vemos en Lavoisier a un científico que hizo notables aportes a la biología, ellos son sobre todo sus trabajos relacionados con la respiración animal, que era considerada por él como una combustión muy lenta, donde participaba como combustible el carbono y el hidrógeno procedentes de la alimentación, y como comburente, el oxígeno, proveniente del aire.

Como conclusión de este aparte, queremos resaltar que si bien puede verse en el quehacer de Lavoisier una yuxtaposición de actividades en diferentes campos del saber, asimismo podríamos comprenderlo como la integración de diferentes saberes y procedimientos que confluyeron en sus investigaciones químicas. Esta situación puede deberse, en parte, a la utilización de aspectos conceptuales y procedimentales cimentados en la física, para dar cuenta de una nueva problemática no resoluble desde esa disciplina. También puede inferirse que Lavoisier utilizó las "artes de la abogacía" para defender sus ideas científicas. No obstante, hay que precisar que él también ideó y puso a prueba técnicas y conceptos que podríamos denominar como netamente químicos, pero esto será discutido en la siguiente sección.

\section{Lavoisier como decapitador del flogisto. 0 la discontinuidad en la historia de las ciencias}

El paso de una teoría a otra no se hace tanto por añadidura de conceptos, sino por la creación de nuevas entidades que pueden resultar contradictorias con las anteriores. Aquí retomaremos el ejemplo de cómo la doctrina del flogisto fue desterrada de la ciencia por parte de la teoría del oxígeno. Antes de mirar la manera en que se llevó a cabo esa revolución química, es necesario que recordemos algunos de los postulados de la combustión explicada desde el flogisto.

Georg Stahl fue un médico alemán que vivió entre los siglos XVII y XVIII, y postuló la idea de un principio de inflamabilidad al que denominó flogisto, que en griego significa arder. Con su doctrina, Stahl estaba poniendo fin a una tradición alquimista para dar origen, según él, a una verdadera química científica, lo que queda de manifiesto en estas líneas escritas en 1723:

La química ha sido durante más de doscientos años dominio exclusivo de los charlatanes, que han causado una infinidad de víctimas (...) Actualmente algunas personas han comenzado a ocuparse seriamente 
de esta ciencia. No debe sorprendernos que su número sea pequeño. Era natural que los impostores, las falsas promesas de los fabricantes de oro, los supuestos misterios, los remedios universales y las preparaciones farmacéuticas, a menudo nocivas, de los alquimistas convirtieron la química en algo odioso a las personas honestas y sensibles, y suscitaron en ellas un sentimiento de disgusto provocado por un saber caracterizado por el fraude y por la impostura (citado por Rossi, 1998: 158).

Para Stahl, hay una clara distinción entre la charlatanería propia de los alquimistas y el discurso argumentado característico de la ciencia. Sin embargo, la teoría que él propone no rompe con la tradición, ya que sigue conservando los cuatro elementos aristotélicos, con los que da cuenta de la composición y transformaciones de la materia. Es decir, que las concepciones de Stahl siguen siendo básicamente esencialistas y substancialistas.

Para poder llevar a cabo su proyecto de separar la química de la alquimia, pero además para distanciarse del mecanicismo newtoniano, Stahl recurre a sus maestros y, al parecer, el flogisto es una modificación de la segunda tierra de Becher y del azufre o principio de combustión de Paracelso. Las tierras de Becher son: vitrificable, combustible y mercurial, las que se corresponden con tres substancias: sal, azufre y mercurio, que a su vez tienen una afinidad con tres principios: de fijación o solidificación, de combustibilidad y de volatilidad (Estany, 1990: 23). Instaura así una nueva forma de explicar los cambios en la materia, pero cimentando sus bases en principios difíciles de observar y de medir.
Sin embargo, el flogisto no es la única idea de la teoría de Stahl, lo que sucede es que esta es la que ha alcanzado más renombre, sobre todo porque se considera como la idea central de la química anterior a Lavoisier:

Parte de su relevancia proviene del hecho de que la teoría del flogisto puso el énfasis de su explicación en los fenómenos relacionados con la combustión, y fue precisamente la investigación de la combustión la que llevó a Lavoisier al descubrimiento del oxígeno (Estany, 1990: 22).

Según Estany (1990), además de la doctrina del flogisto, la obra de Stahl contiene otras tres teorías:

Teoría de la materia: su idea fundamental es que las masas materiales poseen propiedades específicas que permiten reconocerlas y prever sus reacciones. Estas masas materiales se diferencian por su composición y no pueden ser divididas al infinito.

Teoría de la composición: trata de explicar las combinaciones entre los principios elementales, la descomposición y el desplazamiento de un cuerpo por otro.

Teoría de las sales: pretende caracterizar la primera tierra de Becher, es decir la vitrificable.

Por su parte, la idea del flogisto encaja perfectamente en la idea que Stahl tiene de la química como "el arte de descomponer cuerpos compuestos en sus principios básicos y recomponerlos de nuevo":

La química, llamada otras veces alquimia y espagírica, es el arte de disolver los cuerpos, tanto mixtos como compuestos o incluso los agregados, en sus principios elementales, o bien de combinarlos en tales a partir de 
los principios elementales. Tiene por sujeto todos los cuerpos mixtos y compuestos que son solubles y combinables. Tiene por objeto la disolución y combinación mismas, o la corrupción y generación: y de la misma manera que no se alcanza el fin sin los medios, así también corresponden a esta ciencia distintas cosas según la diversidad de los sujetos (citado por Estany, 1990: 23-24).

Vemos en las palabras de Stahl algo que se hizo muy evidente en sus discípulos: la idea de la combustión como análoga a la disolución. Recuérdese que para Priestley, por ejemplo, la combustión consistía en que un cuerpo saturado de flogisto, lo perdía al quemarse y si el aire estaba desflogisticado este se saturaba de dicho principio. Cuando el aire se flogisticaba se detenía la combustión, porque él no podía disolver más flogisto.

La teoría del flogisto puede simplificarse como sigue: este es un principio ontológico imponderable que se encuentra en todos los cuerpos combustibles; cuanto más flogisto posea un cuerpo, mayor será su capacidad para combustir. Además, el flogisto puede pasar de un cuerpo que lo posee en gran cantidad a otro que lo tiene menos, o no lo posee en absoluto. Y por último, el aire puede saturarse de flogisto durante la combustión, y cuando ello ocurre ya no hay posibilidad de que continúe dicho proceso. A la luz de nuestros conocimientos actuales, este tipo de argumentación sería relativamente fácil de refutar; sin embargo, esta teoría estuvo vigente durante aproximadamente un siglo.

A pesar de ser un ente imponderable, Stahl caracterizó al flogisto de la siguiente manera (Estany, 1990: 30): es considerado como el principio de la combustibilidad, que no se debe confundir con la llama o el fuego-material. No se puede coger (es inasible), es indisoluble ${ }^{3}$, y compone en mayor o menor proporción todos los cuerpos susceptibles de ser consumidos por el fuego. En su Tratado sobre el azufre, Stahl le confirió las siguientes propiedades al flogisto o principio-fuego (Citado por Estany, 1990, 30):

La primera cosa a examinar en relación con el principio azufroso es:

1. Su propiedad relativa al fuego.

2. La propiedad que tiene de colorear.

3. Su combinación íntima con otras substancias sutiles.

4. La manera de comportarse en relación con el agua y la humedad.

5. Su sorprendente división y atenuación.

6. Su naturaleza, sea en estado sólido, sea en estado fluido.

7. Dónde se encuentra.

Podríamos preguntarnos cómo una explicación que se basa en un principio muy difícil de ser observado y cuyas propiedades no puedan demostrarse por medio de la experimentación, haya podido permanecer en la tradición científica durante un periodo tan largo. A lo que puede responderse argumentando que no hubo una teoría que explicara mejor la combustión, hasta que Lavoisier propuso la explicación del oxígeno ${ }^{4}$.

3 Pero como vimos, esta idea cambió en algunos de sus discípulos.

4 No desconocemos la posibilidad de que los seguidores de la doctrina del flogisto hayan medido este principio y hayan ideado pruebas experimentales que confirmaran sus afirmaciones. Es decir, no 
Quizás el punto débil de la teoría del flogisto consistió en su incapacidad para explicar por qué había un aumento en el peso de los metales después de la calcinación. Ello se argumentaba diciendo que el flogisto poseía un principio levitatorio, que era contrario a la fuerza de gravedad o, en otros términos, que poseía un peso negativo. También se argumentaba que la materia del fuego remplazaba al flogisto que abandonaba al cuerpo durante la combustión, lo que implicaba que esta tuviera un peso muy elevado. Sin embargo, el sentido común nos conduce a pensar en el fuego ${ }^{5}$ más como un fluido liviano que como un cuerpo sólido y muy pesado:

Otro problema consistió en explicar por qué cesa la combustión en un recipiente cerrado, situación que se explica diciendo que el aire pone en movimiento las partículas del flogisto y cuando este movimiento alcanza una velocidad suficiente se desprende el flogisto del cuerpo (Mosquera, Mora y García, 2003: 117).

Es precisamente a partir de estas observaciones, con respecto al aumento del peso de los metales tras la calcinación y la supuesta flogistización del aire durante la combustión, que Lavoisier entró en conflicto con la doctrina del flogisto, pero no lo hizo abiertamente, sino a par-

asumimos que dicha doctrina estuviera cimentada solamente en posturas metafísicas. Lo que queremos reiterar es que la teoría del oxígeno demostró ser más apropiada para dar cuenta del fenómeno de la combustión.

5 Como ha señalado Bachelard en su Psicoanálisis del fuego, este aún sigue siendo un problema de conocimiento, y en los cursos de química dicha problemática se ha omitido u obviado, en vez de ser abordada y superada. Al respecto véase el texto de Orozco (1996). tir de un plan que hoy podríamos asumir como perfectamente diseñado.

Según Bensaude-Vincent (1998), los historiadores contemporáneos distinguen en este episodio la estructura de una revolución científica que se caracteriza por síntomas de crisis, aparición de un nuevo paradigma y división y conflicto en el seno de una comunidad científica ${ }^{6}$. Dicha revolución lavoisieriana culminó con la publicación del Tratado elemental de química, precisamente el mismo año en que comenzó la Revolución Francesa, en 1789. Pero veamos de qué modo se llevó a cabo esta importante ruptura en el saber científico.

En muchos lugares se ha afirmado que la revolución de Lavoisier de debió sobre todo al uso de la balanza, mediante la cual pesó los reactivos, los productos y los implementos usados en una combustión, antes y después del proceso. Sin embargo, esto no es cierto del todo, pues ya se usaba este instrumento en las experiencias químicas, aunque no se hacía desde la perspectiva en que lo hizo Lavoisier. Es decir, que más que el uso de la balanza en sí, lo que Lavoisier introdujo en la química fue una nueva forma de plantear y de asumir los problemas relativos a los cambios de masa tras una combustión. No es el uso del instrumento per se, sino la teoría que guía su uso lo que cuenta a la hora de hablar de una innovación metodológica. Desde ese momento, la balanza es una especie de juez experimental, en especial el estudio de los gases:

6 Todo ello característico del lenguaje de Kuhn para dar cuenta de las revoluciones científicas. Afirmaciones que cuestionaremos, en parte, en el apartado de reflexiones finales. 
Su utilización se impone junto con la de la caja neumática, un aparato cerrado que sirve para recoger los gases, y el gasómetro, que permite medir el volumen de los mismos. Joseph Black, Carl Sheele, Henry Cavendish y Lavoisier apelan a toda la habilidad y el ingenio de los artesanos de su país para obtener balanzas cada vez más precisas y perfectas (BensaudeVincent, 1998: 415).

Lavoisier pesa todo antes y después del experimento, lo que ha sugerido su paternidad de la ley de la conservación de la masa, pero al parecer no es de su autoría. Sin embargo, este principio orientó su quehacer en el laboratorio y le permitió emitir las conclusiones que llevaron al derrumbamiento de la teoría del flogisto.

Lavoisier decidió colocar en un sobre lacrado los resultados obtenidos en sus primeros experimentos sobre la combustión, donde daba cuenta del aumento del peso de las sustancias después de dicho proceso. Este sobre fue entregado a la Academia el primero de noviembre de 1772 y solo fue abierto el 5 de mayo del año siguiente, cuando sus trabajos y conclusiones estaban más avanzados. Dentro del sobre, Lavoisier consignó lo siguiente:

Hace unos ocho días descubrí que al quemar azufre, el peso de éste, en lugar de disminuir, aumentaba; es decir que de una libra de azufre podíamos obtener más de una libra de ácido vitriólico, sin tener en cuenta la humedad del aire; lo mismo ocurre con el fósforo: este aumento de peso proviene de la cantidad prodigiosa de aire que se fija durante la combustión y que se combina con los vapores. Este descubrimiento, resultado de una serie de experimentos que con- sidero decisivos, me indujo a pensar que lo que observaba en la combustión del azufre y del fósforo podía tener lugar, igualmente, con todos los cuerpos que ganan peso tras la combustión o la calcinación; y me convencí incluso de que el aumento de peso de las cales metálicas respondía a la misma causa. El experimento ha confirmado completamente mis conjeturas; he procedido a reducir litargirio en recipientes cerrados, con el aparato de Hales, y he observado que, en el momento en que la cal se convierte en metal, se desprende una cantidad considerable de aire, y que este aire forma un volumen mil veces superior al de la cantidad de litargirio utilizada. Este descubrimiento me parece uno de los más interesantes desde los de Stahl y, por ello, he considerado que era mi deber asegurarme de su autoría, depositando ante la Academia el presente sobre, para que sea mantenido en secreto hasta que publique mis experimentos (citado por Bensaude-Vincent, 1998: 418).

Vemos en este párrafo que Lavoisier cita a Stahl como un antecesor, pero en realidad lo que está proponiendo es poner de presente que, si bien ha sido uno de los patriarcas de la química, su doctrina carece de coherencia con la experimentación. Es importante que nos centremos en mirar de qué manera los trabajos de Lavoisier permitieron echar a tierra los fundamentos de la doctrina de Stahl. En primer lugar están sus trabajos que llevaron a confrontar la idea de los elementos-principios, los cuales están a la base de la teoría del flogisto y que habían sido heredados de la tradición griega.

Inició con unos trabajos sobre la composición del yeso, o sea, sobre la tierra, 
cuando aún su interés estaba centrado en la geología. Posteriormente, y por encargo de la Academia, hizo estudios sobre el alumbrado público, es decir, sobre el fuego y la combustión, trabajo realizado en 1774. Tres años más tarde realizó un trabajo sobre la composición del agua de los Vosgos ${ }^{7}$. Y posteriormente llevó a cabo una investigación acerca del suministro de agua en París, en el que estudió las relaciones entre el agua y la tierra. En la tradición basada en los elementos-principios se aseguraba que cada uno de ellos podía trasmutarse en alguno de los tres restantes, por lo que no era descabellado afirmar que el agua se convertía en tierra.

Esto parecía ser explicado por la aparición de un residuo sólido en el agua de París, idea que fue refutada por Lavoisier al demostrar que después de 101 días de ebullición, el peso de un volumen de agua se mantiene constante, mientras que el recipiente pierde un peso igual al de los residuos sólidos que aparecen en el fondo. El agua no se transforma en tierra, sino que disuelve cierta parte del recipiente, que luego se sedimenta:

Así mismo, según las notas manuscritas en 1776 y 1778, Lavoisier trabaja sobre las relaciones entre el agua y el fuego, y, tras diversas lecturas, adopta la idea de que todas las sustancias pueden existir en los tres estados (sólido, líquido o aeriforme) dependiendo de la cantidad de materia del fuego que se combina. Lavoisier ha examinado, uno por uno, los cuatro elementos (BensaudeVincent, 1998: 420).

7 Cadena montañosa situada al nordeste de Francia, donde existen yacimientos minerales, principalmente de hulla y petróleo.
Es decir que ha sustituido la tierra por lo sólido, el aire por lo aeriforme, el agua por lo líquido y el fuego ahora es el responsable del estado, de acuerdo con su cantidad.

Lavoisier inició sus experimentos sobre el flogisto en 1772, a partir de una publicación de Guyton de Morveau, donde explicaba el aumento de peso del plomo durante la calcinación, admitiendo la pérdida de flogisto, que tenía peso negativo. Al parecer, Lavoisier ya había concebido su plan revolucionario, aunque en sus primeros escritos fue muy discreto y no atacó abiertamente las ideas de Stahl y sus seguidores:

La explicación de Lavoisier es opuesta a la precedente: la combustión libera el flogisto contenido en el cuerpo combustible, para Lavoisier se trata de una combinación con el aire; la fuente de calor ya no se encuentra en el combustible (flogisto), sino en el aire (calórico). Ello supone la inversión del papel del calor: para Stahl el calor, al fijarse, provoca una combinación o una condensación. Para Lavoisier, provoca una expansión, incluso una desagragación (Bensaude-Vincent, 1998: 421).

Podríamos decir que el trabajo de Lavoisier consistió en erradicar la teoría del flogisto, inventando una nueva ontología, en gran parte opuesta a la anterior. Si bien se le ha refutado el hecho de querer erradicar los elementos-principios y mantener el nombre de elemento, de sustituir el flogisto por el calórico y de asumir que el oxígeno hace parte de todos los ácidos, también es cierto que:

- El elemento ya no lo define como un concepto metafísico, sino como lo obtenido mediante el análisis experimental. 
- El calórico no es equiparable al flogisto, ya que este sí es ponderable mediante el termómetro, mientras que el flogisto escapa a una medición de ese estilo.

- A pesar de que el oxígeno no es un principio de la acidez ${ }^{8}$, sí es la base de la mayoría de las combustiones. Es decir, que actualmente este elemento no juega un papel en la teoría de los ácidos, pero sigue siendo el ingrediente más importante a la hora de explicar la combustión.

De acuerdo con Bensaude-Vincent (1990), la campaña revolucionaria de Lavoisier se llevó a cabo en tres etapas, que resumiremos enseguida.

El proyecto de Lavoisier comienza en 1783, cuando demuestra que el agua está conformada por dos elementos, es decir, echa al piso el último de los elementos-principios. Dichas experiencias fueron de síntesis y no de análisis. Del mismo modo, a partir de su memoria Reflexiones acerca del flogisto, empieza a utilizar un tono más duro y deja la pru-

8 Etimológicamente hablando, y desde una perspectiva contemporánea, Lavoisier no descubrió el oxígeno, ya que ello significa formador de ácidos, nombre que merecería más nuestro actual hidrógeno. De hecho, el oxígeno tendría el mismo derecho de llamarse hidrógeno, porque también es formador de agua. Sin embargo hay que precisar que si Lavoisier denominó al oxígeno de esta manera es porque él consideraba que en toda combustión (obviamente en presencia de oxígeno) se formaban ácidos. En ese sentido no sería ilógico que hubiese utilizado tal nominación. Por ejemplo, él creía que si se quemaba fósforo en presencia de aire eminentemente respirable, se produciría ácido fosfórico. Por lo tanto, si Lavoisier creía que el oxígeno participaba en la formación de los ácidos (como una condición), y que aquel podría entrar a hacer parte de la composición de estos, entonces es posible comprender su propuesta de bautizar así a dicho elemento. dencia a un lado, ya que es consciente de que ha abonado muy bien el terreno para cimentar su revolución. Prueba de ello está en una de las primeras páginas de la memoria mencionada:

Ruego a mis lectores, desde el comienzo de esta memoria, que se despojen, en la medida de lo posible, de cualquier tipo de prejuicio: que no vean en los hechos más de lo que presentan, que eliminen todo lo que el razonamiento ha dado por supuesto, que se trasladen a los tiempos anteriores a Stahl, y que olviden, por un instante, de ser posible, la existencia de su teoría (Lavoisier, 1956 [1783]: 122).

Podemos apreciar la manera en que invita a sus lectores a borrar el pasado, a que se sumerjan en una amnesia colectiva, ya que de todas maneras quiere hacer desaparecer la teoría de Stahl. En este mismo escrito, Lavoisier comenta que Stahl asume el flogisto como un principio terroso, y que, por tanto, pesa. Sin embargo, su teoría no explicaba un hecho verificado por Boyle, que "todos los cuerpos combustibles aumentan de peso cuando se queman y calcinan" (Lavoisier, 1956 [1783], 124), y según la teoría del flogisto, los cuerpos al quemarse pierden este principio, lo que les llevaría a perder peso, pero en vez de perder, aumentan.

Una vez que ha preparado los experimentos sobre la síntesis del agua y ha superado los problemas logísticos, como la consecución de grandes cantidades de hidrógeno, Lavoisier termina su primera etapa revolucionaria: realiza la síntesis del agua, en presencia de varios sabios y nobles de su época, lo que le permite rodearse de aliados, dentro de los que se destacan Berthollet, Fourcroy y Guyton de Morveau. 
La segunda etapa inicia cuando, ya rodeado de aliados, emprende un cambio en el lenguaje de la química, para dotarla de las características de una ciencia universal, en la cual se hable un idioma entendible por todo el mundo. Lavoisier, inspirado en la importancia del lenguaje, a partir de la lectura de los textos del abate de Condillac, inicia esta nueva tarea:

La supresión de los nombres usados corrientemente y la construcción de una lengua artificial, basada únicamente en la teoría de Lavoisier, suponía la desvinculación con el pasado. Mejor aún: un renacimiento a través del bautismo (Bensaude-Vincent, 1998: 423).

Esta empresa culmina en 1787 con la publicación del Método de nomenclatura química, cuyos autores son Lavoisier, Berthollet, Fourcroy y Guyton de Morveau. Sin embargo, a pesar de ser un trabajo colectivo, Lavoisier se reserva el papel de director. Podemos afirmar que esta propuesta de cambiar los nombres de las sustancias:

Es mas bien un "método de designar" que una nomenclatura. El principio básico es una lógica de la composición: crear un alfabeto de palabras simples para designar sustancias simples; luego designar las sustancias compuestas con nombres compuestos, formados por yuxtaposición de nombres simples (Bensaude-Vincent, 1998: 423).

Con la creación de la nueva nomenclatura, Lavoisier lleva a cabo una doble ruptura; de un lado, con el lenguaje antiguo, usado durante más de un siglo. De otro lado, hay una ruptura entre el lenguaje químico de los académicos y el de la química artesanal practicada por dro- gueros y perfumistas. Vemos pues, que se propone institucionalizar el lenguaje químico, distanciándolo del vulgo.

La tercera y última etapa de la revolución química es la publicación del Tratado elemental de química, que es presentado por Lavoisier como una continuación lógica de sus trabajos sobre nomenclatura. Una vez desterrado el pasado, debe asegurarse de que su obra sea difundida: hay que reclutar a los principiantes. Sin duda alguna, las etapas precedentes le abrieron el camino para que se erigiera como nuevo fundador de la química: como ha borrado con el pasado, la mejor manera de asegurarse que su obra sea difundida y aceptada es llegar al público joven, que no tiene ningunos prejuicios $\mathrm{y}$, por tanto, puede aprender esta ciencia más fácilmente.

En el Discurso preliminar de su Tratado, Lavoisier hace declaraciones importantes que es bueno tener de presente. En primer lugar, se va lanza en ristre contra los elementos aristotélicos:

He de advertir aquí que la manía que tenemos de que todos los cuerpos naturales se compongan únicamente de tres o cuatro elementos se debe a un prejuicio heredado de los filósofos griegos. Admitir que cuatro elementos componen todos los cuerpos conocidos sólo por la diversidad de sus proporciones, es una mera conjetura imaginada mucho antes de que se tuviesen las primeras nociones de la física experimental y de la química (Lavoisier, 1982 [1789]: 10).

Una vez que refuta esta definición de elemento, procura emitir la suya propia, lo que es un aspecto bastante importante del proceder de Lavoisier:

Todo lo que puede decirse sobre el número y la naturaleza de los ele- 
mentos se reduce, en mi opinión, a puras discusiones metafísicas: sólo se intenta resolver problemas indeterminados susceptibles de infinitas soluciones, ninguna de las cuales, con toda probabilidad, será acorde con la naturaleza. Me contentaré, pues, con decir que si por el nombre de elementos queremos designar a las moléculas simples e indivisibles que componen los cuerpos, es probable que los ignoremos; pero si, por el contrario, unimos al nombre de elementos o principios de los cuerpos, la idea del último término al que se puede llegar por vía analítica, entonces todas las sustancias que hasta ahora no hemos podido descomponer por cualquier medio serán para nosotros otros tantos elementos; con esto no queremos asegurar que los cuerpos que consideramos como simples no se hallen compuestos por dos o mayor número de principios, sino que como nunca se ha logrado separarlos, o, mejor dicho, faltándonos los métodos para hacerlo, debemos considerarlos cuerpos simples y no compuestos hasta que la experiencia y la observación no demuestren lo contrario (Lavoisier, 1982 [1789]: 11).

Igualmente se encuentra en este Discurso preliminar una invitación a romper radicalmente con toda la historia pasada, es decir, Lavoisier se propone fundar un nuevo saber:

Quizá se me censuraría con más fundamento, el que no dé en la obra que presento al público ninguna relación histórica de las opiniones de los que me han precedido y sólo muestre las mías sin examinar las de los demás. De aquí resulta que no he dado a mis colegas, y menos a los extranjeros, la justicia que deseaba rendirles; pero ruego al lector considere que si se acumulan citas en una obra elemental, si nos detenemos en prolijas consideraciones sobre la historia de la ciencia y de las aportaciones de los que la han cultivado, se perdería de vista el objeto principal marcado y se elaboraría una obra de lectura fastidiosa para los principiantes. No es un tratado elemental el lugar adecuado para hacer la historia de la ciencia ni del espíritu humano; en él sólo se debe buscar la facilidad y claridad, procurando descartar todo lo que podría tender a distraer la atención. Este es un camino que se ha de ir allanando continuamente sin dejar pervivir ningún obstáculo que pueda ocasionar el menor retraso (Lavoisier, 1982 [1789]: 16).

Vemos que aquí lo elemental tiene dos connotaciones; de un lado, está la definición de elemento analítico, en oposición al elemento escencialista. Y, de otro lado, lo elemental se concibe como el primer escalón para llegar al conocimiento químico. Se construyen los cuerpos a partir de los elementos químicos y se construye el conocimiento a partir de los conceptos elementales.

Dejemos de lado la química, por un momento, para observar la manera en que el conflicto entre Lavoisier y Stahl va más allá de la confrontación entre oxígeno y flogisto. Esta ruptura también se halla en la concepción de lo vivo. Para Stahl la doctrina filosófica a defender es el animismo, mientras que Lavoisier se inscribe en el mecanicismo.

Según Radl (1988), el vitalismo (o, más precisamente, el animismo) como doctrina filosófica, se presenta por primera vez en las concepciones de Stahl. Dichos planteamientos se desarrollaron como contraposición al mecanicismo surgido de los trabajos de Newton. Para Stahl, 
el organismo es totalmente opuesto al mecanismo, ya que en el primero tienen lugar los movimientos, mientras que en el segundo ellos son producidos.

La teoría del alma (ánima) constituye la médula de la filosofía natural de Stahl. También en la biología aristotélica desempeña el concepto del alma, de la psique, el papel más importante. Pero Stahl no fue capaz de remontarse al audaz concepto aristotélico del alma. Mientras en Aristóteles el alma significa la médula del organismo, de su forma y de sus fuerzas, Stahl se dejó influir por el concepto cristiano y por la concepción cristiana del alma, como principio inmaterial, consciente, que planea sobre la máquina corpórea. Por eso se llama su teoría animismo. Stahl conserva el dualismo cartesiano del espíritu y el cuerpo. Enseña que el alma no puede nada sin el cuerpo; no puede percibir nada, ni por tanto conocer nada sin los sensorios corporales; no puede realizar su voluntad sin el servicio de los órganos corporales. Por eso es necesario el cuerpo; lo es como medio que hace posible las intenciones del alma (Radl, 1988: 210).

Apreciamos en la doctrina de Stahl, no solo el mantenimiento de los cuatro elementos, sino también el concepto de alma heredado de la tradición griega y luego reelaborado por el cristianismo. El alma es como esa fuerza vital, que no se puede ver ni medir, pero que le confiere la propiedad de vivo a los seres animados $^{9}$. Son bastantes las similitudes entre el concepto de alma y el de flogisto, con

9 Desde la doctrina del animismo se asumía a lo viviente como dotado de un ánima, y lo inerte, carente de ella, de ahí el significado de ser animado e inanimado. la diferencia de que el primero explica lo vivo y el segundo las combustiones.

Podemos decir, entonces, que el mecanicismo también tiene varias acepciones, dependiendo del contexto desde el cual se hable. El mecanicismo newtoniano asumía que todo está compuesto de partículas inertes que se mueven según la fuerza que se aplique sobre ellas. Así como en el cielo hay una máquina que permite el movimiento de los astros, del mismo modo en el mundo microscópico hay ciertas condiciones que permiten los engranajes entre las partículas y así facilitar el movimiento. Stahl reaccionó contra este tipo de mecanicismo:

Pero en contra del programa mecanicista y newtoniano, basado en la absoluta homogeneidad y que corría el riesgo de llevar a la investigación a un callejón sin salida, advertía precisamente Stahl de la necesidad de retornar a la química de los principios y a los elementos de la tradición esencialista. Y aún más: Stahl admiraba profundamente la Physica subterranea de Joachim Becher (Rossi, 1998: 158).

Con este título, Stahl hizo reeditar la obra de su maestro en 1669. En dicho trabajo se hallan ciertas afirmaciones que pueden entenderse como un retorno al paracelcismo, ya que se asume:

La idea de que el estudio de la naturaleza debe comenzar con una explicación del relato mosaico de la creación; la analogía macrocosmosmicrocosmos; el paralelismo entre vegetales y animales; la creencia en la generación espontánea. La tesis de que los metales "crecen" en las entrañas de la Tierra; por último, el paralelismo entre la perpetua y eterna circulación que se produce en el cosmos y la destilación química (Rossi, 1998: 159). 
Es decir que todo está impregnado de vida, no hay una clara diferenciación entre el mundo vivo y el inerte. De igual manera que el flogisto puede pasar de un cuerpo a otro, el principio vital puede alojarse en los minerales que crecen, o la materia inerte espontáneamente puede ser activada por este principio vital.

Por su parte, Lavoisier concebía lo vivo, principalmente los animales, desde el punto de vista mecanicista, que sostiene la total identidad entre un organismo y una máquina. Él trasladó sus investigaciones sobre la combustión a la respiración animal. De hecho, para Lavoisier la respiración no es otra cosa que una combustión, pero más lenta. Veamos, entonces, cómo desplazó sus experimentos hacía la fisiología animal.

Encerró en un aparato 50 pulgadas cúbicas de aire común y 4 onzas de mercurio muy puro, que sometió a calcinación. Al cabo de algunos días, aparecieron puntos rojos sobre el metal, que fueron aumentando con el paso del tiempo. Luego, al terminar el experimento, notó que había una disminución en el volumen de aire original, equivalente a la cal de mercurio aparecida. El aire restante tenía la propiedad de apagar una vela, no servía para respirar y no precipitaba el agua de cal. Es decir que el mercurio se había combinado con la mejor parte del aire, la que en realidad sirve para respirar.

Posteriormente hizo el experimento contrario; redujo la cal de mercurio y obtuvo aire respirable, que mezcló con el mefítico (el que había quedado en el experimento anterior) y produjo nuevamente aire común. De ahí pudo deducir que el aire está formado por "un quinto de aire eminentemente respirable y por cuatro quintos de una mofeta in- capaz de permitir la respiración de los animales"(Lavoisier, citado por Giordan et ál., 1988: 48).

Lavoisier repitió su primer experimento, pero en vez de calentar el mercurio, introdujo un gorrión, que al cabo de 55 minutos murió. Inmediatamente introdujo otro, pero este solo sobrevivió unos instantes. La diferencia entre los dos experimentos se halló en el hecho de que el aire producto de la respiración, además de apagar las velas, sí precipitaba el agua de cal y no hubo un cambio sustancial entre el volumen de aire inicial y el final.

Para Lavoisier es posible que el oxígeno que entra en el cuerpo del animal se absorba por la sangre y sea remplazado por gas carbónico (aire fijado), mientras que el aire mefítico (nitrógeno) no sea transformado en la respiración y sea pasivo con respecto a esta. Lavoisier trabajó con Seguin sobre la respiración animal y en sus conclusiones puede verse una concepción del animal como máquina: En la combustión y en la respiración se consume oxígeno y se produce gas carbónico y calórico, pero en el animal, es la sangre misma la que suministra el combustible. De ahí que Lavoisier y Seguin afirmen que: "Si los animales no recuperaran, habitualmente mediante los alimentos, lo que pierden por la respiración, pronto faltaría aceite a la lámpara, y el animal perecería, igual que una lámpara se extingue por falta de sustento" (citados por Giordan et ál., 1988: 50).

Una vez que Lavoisier y Seguin han encontrado las interrelaciones entre respiración y nutrición, deben proponer un mecanismo que explique la liberación de calor, para evitar el recalentamiento del animal. Por ello afirman la necesidad 
de la transpiración, puesto que esta "facilita la liberación de cierta cantidad de calórico... y, en consecuencia, impide que el individuo, merced al continuo enfriamiento que produce, no alcance un grado de temperatura superior al marcado por la naturaleza" (citados por Giordan et ál., 1988: 50).

A partir de 1780, Lavoisier inicia sus trabajos sobre el calor junto con Laplace. Crean aparatos de calorimetría para medir el desprendimiento de calor producido por la respiración animal, y asumen que esta cantidad es la causa de la conservación del calor del organismo. Y asumen también que la respiración es una combustión pero muy lenta, donde no hay evidencia del desprendimiento de luz y fuego, ya que estos son absorbidos por la humedad de los órganos. Dicho proceso de combustión tiene lugar en los pulmones y la sangre lleva el calor a todo el cuerpo del animal, de lo que se deduce que:

(...) el aire que respiramos tiene dos objetivos, igualmente necesarios para nuestra conservación: retira de la sangre la base de aire fijo cuya sobreabundancia sería muy perjudicial; y el calor que esta combustión libera en los pulmones repara la pérdida continua de calor que sufrimos debido a la atmósfera y a los objetos que nos rodean (Lavoisier, citado por Giordan et ál., 1988: 50).

Posteriormente y a partir de los experimentos sobre calorimetría, Laplace y Lavoisier descubren que durante la respiración el calor liberado es mayor de lo que se esperaría. Por tanto, sugieren que el carbón no es el único combustible, sino que además interviene el hidrógeno, ambos provenientes de los alimentos. Al asumir la participación del hidrógeno en la respiración, pueden además explicar la aparición de agua como producto de esta.

Para finalizar este aparte, veamos algunas de las conclusiones que emitió Lavoisier, a partir de sus experimentos sobre la respiración, donde se evidencian sus concepciones mecanicistas opuestas al animismo de Stahl:

La máquina animal está gobernada fundamentalmente por tres reguladores principales: la respiración que consume hidrógeno y carbono y proporciona calórico; la transpiración que aumenta o disminuye, según se necesite retirar más o menos calórico; y, por último, la digestión, que suministra a la sangre lo que pierde por la respiración y la transpiración (Lavoisier, citado por Giordan et ál., 1988: 52).

\section{Lavoisier venerado y decapitado. 0 la estrecha relación entre ciencia y sociedad}

La última característica de la historia de la ciencia que pretendemos resaltar en este trabajo consiste en dar cuenta de cómo la actividad científica no puede ser ajena a un contexto sociocultural, pero de igual manera se quiere evidenciar que las sociedades no son indiferentes al quehacer de los científicos.

En el caso de Lavoisier nos encontramos con una paradoja, que ya ha sido resaltada por varios autores: el padre de la química fue victorioso en la revolución científica que llevó a cabo, pero otra revolución, la Francesa, lo condenó a muerte en la guillotina; aquel que dedicó varios años de su vida para dejar sin cabeza a la doctrina del flogisto, perdió la suya en un instante, después de haber sido condenado por traición a la patria. 
Pero las paradojas no terminan allí: su revolución química, que le dio gloria, culminó exactamente el mismo año en que se inició la Revolución que le arrebató la vida. Según las historias que se han escrito de Lavoisier, el hecho de haber sido recaudador de impuestos del viejo régimen, llevó a ser considerado como traidor por el nuevo orden político que estaba imponiéndose en Francia.

Uno de los aspectos que queremos resaltar es que, entre una revolución científica y una revolución política existen muchas similitudes. Esto queda de manifiesto en hechos como los siguientes: en ambos tipos de revolución se quiere acabar el viejo orden para poder instaurar uno totalmente nuevo; el nuevo paradigma no crea sobre las bases de su antecesor, sino a partir de sus ruinas. Igualmente, en los dos hay un cambio del lenguaje, y así como Lavoisier quiso hacer olvidar los nombres antiguos, para cimentar un nuevo idioma, de igual manera la Revolución Francesa se esforzó por cambiar, entre otras cosas, el nombre de los meses, empezó a contar el tiempo nuevamente y racionalizó los pesos y las medidas, los que ahora estarían basados en aspectos meramente naturales; ya no se hablaría del pie, sino del metro.

La revolución francesa suprimió el antiguo calendario y empezó su Año I el 22 de septiembre de 1792, año de la fundación de la República, igualmente

Los meses ya no llevarían nombres de dioses o emperadores romanos, sino que registrarían el paso natural de las estaciones (tal era el caso de brumaire, brumoso; ventose, ventoso; germinal, germinal), parte de los meses de julio y agosto, originalmente nombrados en honor de dos césares despóticos, serían sustituidos por el mes thermidor (termidor) (Gould, 1993: 324).

Hay otro aspecto que creemos necesario resaltar en este aparte y es el hecho de que la actividad científica no es obra de un hombre aislado, sino que el motor de la ciencia es precisamente su carácter colectivo. Si bien algunos textos privilegian la individualidad en la labor científica y encontramos declaraciones como la siguiente:

Esta teoría no es pues, como he oído decir, la teoría de los químicos franceses, es mi teoría, y es una propiedad que reclamo ante mis contemporáneos y para la posteridad (Lavoisier, citado por BensaudeVincent, 1998: 430).

Aunque esta frase fue pronunciada por el propio Lavoisier, también encontramos de su parte otro tipo de afirmaciones:

Los químicos se darán cuenta fácilmente de que en la primera parte de esta obra sólo me he servido de experimentos propios. Si alguna vez he utilizado las experiencias o las opiniones de Berthollet, Fourcroy, Laplace, Monge y de todos aquellos que, en general, adoptaron los mismos principios que yo, y haya olvidado citarlos, se debe a que la costumbre de vivir juntos, de comunicarnos nuestras ideas, nuestras observaciones, nuestros puntos de vista, ha establecido entre nosotros una especie de comunidad de opiniones, donde con frecuencia es difícil, incluso a nosotros mismos, distinguir lo que aportó cada uno (Lavoisier, 1982 [1789]: 17).

A nuestro modo de ver, la actividad científica se caracteriza más por la se- 
gunda afirmación de Lavoisier, que por la primera.

Por último, queremos mencionar otro aspecto de la vida de Lavoisier, y es que no podemos separar su actividad como recaudador de impuestos de su actividad científica: cuando iba a una provincia para llevar a cabo su labor de asentista general, realizaba, además, conferencias sobre aspectos científicos en la Academia Local. Su trabajo estaba repartido en las diferentes facetas de su vida, pero al parecer el dinero que ganaba como recaudador lo invertía en parte para costear algunos de sus experimentos. De hecho, nunca pensó en abandonar los cargos que ocupaba y solo lo hizo cuando la Revolución suprimió las instituciones a las que pertenecía: $\mathrm{La}$ Academia y la Ferme Génerale, instituciones que no solo habían sido creadas por el antiguo régimen, sino que habían sido desarrolladas por este.

Lavoisier tenía un horario muy estricto durante la semana, en el cual combinaba perfectamente todas sus labores: de 6 a 8 realizaba investigaciones científicas, a lo largo del día desempeñaba sus funciones administrativas y académicas, nuevamente hacía investigación de 19 a 22 y, además le dedicaba un día entero de la semana para la actividad científica, a este día le dio el nombre de jour de bonheur (día de felicidad) (Gould, 1993: 333).

Quisiéramos cerrar esta sección con la que quizá fue la última frase escrita de Lavoisier, momentos antes de su muerte. Dicha frase proviene de una carta que le escribió a un primo suyo:

He tenido una vida bastante larga, por encima de todo una vida muy feliz, y pienso que seré recordado con cierta pesadumbre y que quizá dejaré algu- na reputación tras de mí. ¿Qué más puedo pedir? Los acontecimientos en los que me veo envuelto me salvarán probablemente de los problemas de la ancianidad. Moriré en plena posesión de mis facultades (Lavoisier, citado por Gould, 1993: 332).

\section{Reflexiones finales}

Hemos desarrollado tres aspectos que consideramos de suma importancia para dar cuenta de un modelo de la historia de las ciencias, los cuales nos han permitido reconocer cómo se ha transformado el quehacer científico desde los siglos XVII y XVIII, cambio que está marcado por un proceso de diferenciación que ha posibilitado la emergencia de disciplinas autónomas como la química. De igual forma, hemos puesto de relieve las complejas e indisolubles relaciones entre ciencia y sociedad. Con respecto a la forma que en se da el cambio científico, hemos hecho una comparación de la revolución lavoisieriana con la Revolución Francesa, lo cual permite encontrar puntos de coincidencia entre las "revoluciones científicas" y las sociales.

No obstante, es de precisar que no asumimos del todo el concepto de revolución científica propuesto por Kuhn, ya que, por ejemplo, no consideramos la total inconmensurabilidad entre paradigmas rivales. Por ello, preferimos optar por la noción de ruptura epistemológica propuesta por algunos autores franceses. Asimismo, asumimos que tales rupturas no pueden ser totales, como lo afirma Canguilhem (2005: 32-33):

Conviene advertir, pues, como indispensable un buen uso de la recurrencia y una educación de la atención a las rupturas. A menudo el investigador de rupturas cree, a la manera 
de Kant, que un saber científico se inaugura con una ruptura única, genial. A menudo también el efecto de ruptura es presentado como un efecto global que afecta la totalidad de una obra científica. Sin embargo, habría que saber detectar en la obra de un mismo personaje histórico rupturas sucesivas o rupturas parciales. Ciertos hilos de una trama teórica pueden ser completamente nuevos, mientras que otros han sido sacados de texturas antiguas. Las revoluciones copernicana y galileana no se hicieron sin conservación de herencias

Hechas estas necesarias aclaraciones, sintetizamos lo expuesto diciendo que las dimensiones de la historia de la

\section{Referencias}

Bensaude-Vincent, B. (1998). Lavoisier: una revolución química. En: Serres, M. (editor). Historia de las ciencias. Madrid: Cátedra.

Canguilhem, G. (2005). [1988]. Ideología y racionalidad en la historia de las ciencias de la vida. Nuevos estudios de historia y de filosofía de las ciencias. Buenos Aires: Amorrortu.

Estany, A. (1990). Modelos de cambio científico. Barcelona: Crítica.

Giordan, A., Raichvarg, D., Drouin, J-M., Gagliardi, R., y Canay, A. M. (1988). Conceptos de biología. Tomo 1: La respiración. Los microbios. El ecosistema. La neurona. Barcelona: Labor.

Gould, S. J. (1993). "Brontosaurus” y la nalga del ministro. Reflexiones sobre historia natural. Barcelona: Crítica.

Lavoisier, A. L. (1956). [1783]. Memorias sobre el oxígeno, el calórico y el flogisto. Introducción y prólogo de Juan M. Muñoz. Buenos Aires: Emecé. ciencia que hemos puesto de presente, pueden devenir en importantes implicaciones didácticas. Por ejemplo, es de esperar que este tipo de reflexiones posibilite a los maestros, en formación inicial o en ejercicio, repensar la manera en que conciben el cambio científico, y remirar las intrincadas relaciones ciencia-sociedad. Reconocer la dimensión histórica de las ciencias puede advertirnos acerca de las problemáticas, hipótesis, alianzas, controversias, negación de tradiciones, fundación de nuevos paradigmas, etc., aspectos muchas veces ausentes en los libros de texto, los programas de estudio y los currículos para la formación de profesores de ciencias. $\mathbf{\Delta}$

Lavoisier, A. L. (1982). [1789]. Tratado elemental de química. Introducción, traducción y notas de Ramón Gago Bohórquez. Madrid: Ediciones Alfaguara.

Mosquera, C. J., Mora, W. M., y García, A. (2003). Conceptos fundamentales de la química y su relación con el desarrollo profesional del profesorado. Bogotá: Centro de Investigaciones y Desarrollo Científico, Universidad Distrital Francisco José de Caldas.

Orozco, J. C. (1996). La dimensión histórico-filosófica y la enseñanza de las ciencias. En: Física y Cultura, 2: Representaciones sobre la ciencia y el conocimiento. Bogotá: Universidad Pedagógica Nacional.

Radl, E. (1988). Historia de las teorías biológicas. Tomo 1. Madrid: Alianza Editorial.

Rossi, P. (1998). El nacimiento de la ciencia moderna en Europa. Barcelona: Crítica. 